\title{
Narrow Notch Width is a Risk Factor for Anterior Cruciate Ligament Injury in the Pediatric Population: A Multicenter Study
}

\author{
Joseph L. Yellin, M.D., Robert L. Parisien, M.D., Nakul S. Talathi, M.D., Ali S. Farooqi, B.A., \\ Mininder S. Kocher, M.D., M.P.H., and Theodore J. Ganley, M.D.
}

\begin{abstract}
Purpose: The purposes of this study were to evaluate the notch width index (NWI) as a risk factor for anterior cruciate ligament (ACL) rupture in the pediatric and adolescent patient population via multicenter analysis and to detect any differences in the NWI among cohorts having sustained either a unilateral or bilateral ACL rupture. Methods: A consecutive series of patients $\leq 19$ years old was enrolled from January 1999 through July 2010 at 2 academic pediatric orthopaedic tertiary-care hospitals. Demographic and anatomic morphology data were collected for 3 cohorts: unilateral ACL ruptures, bilateral ACL ruptures, and a control group. A single blinded reviewer determined notch width measurements via T2 coronal magnetic resonance imaging sequences in a standardized manner, using a previously described technique. Results: Of the 68 patients included for analysis, 22 sustained unilateral ACL rupture, 23 sustained bilateral ruptures, and 23 comprised the control group. There was a statistically significant difference appreciated in direct comparison of the NWI in the bilateral rupture group and the control group, as well as between the unilateral rupture group and the control group. There was no statistically significant difference between the NWI in the unilateral versus the bilateral rupture group. Conclusion: Given the known inconsistencies in the existing literature, our findings provide further support of a narrow NWI as a significant contributing factor to both unilateral and bilateral ACL injury risk in the pediatric and adolescent patient population. Level of Evidence: III, retrospective cohort study.
\end{abstract}

A $s$ the incidence of anterior cruciate ligament (ACL) injury and subsequent reconstruction continues to rise in children and adolescents, numerous studies have attempted to identify independent risk factors and prevention strategies for these significant injuries. ${ }^{1,2}$ Previous studies have established several "nonmodifiable" risk factors for ACL rupture in the young athlete including female sex, generalized ligamentous laxity, and certain morphologic features including increased tibial slope. Several "modifiable"

From the Harvard Combined Orthopaedic Residency Program, Boston, Massachusetts (J.L.Y.); Harvard Medical School, Boston, Massachusetts (R.L.P., M.S.K.); the University of California Los Angeles Orthopaedic Surgery Residency, Los Angeles, California (N.S.T.); the Perelman School of Medicine at the University of Pennsylvania, Philadelphia, Pennsylvania (A.S.F.); Boston Children's Hospital, Boston, Massachusetts (J.L.Y., R.L.P., M.S.K.); and The Children's Hospital of Philadelphia, Philadelphia, Pennsylvania (T.J.G.), U.S.A.

The authors report the following potential conflicts of interest or sources of funding: R.L.P. serves on the American Orthopaedic Society for Sports Medicine: Research Committee and the editorial board for Arthroscopy. M.S.K. is a paid consultant for Best Doctors, OrthoPedics, Ossur, and Smith $\theta$ Nephew; is a board or committee member for Harvard Medical School, the Harvard School of Public Health, the Pediatric Orthopaedic Society of North America, PRISM, and the Steadman Philippon Research Institute; receives IP royalties from risk factors, including neuromuscular control and proprioception, have been identified as well. ${ }^{3-9}$

Although femoral notch width (NW) has been proposed as a risk factor for ACL rupture in the adult population, ${ }^{10}$ the impact of NW on ACL injury risk in adolescents remains controversial. Some authors have hypothesized a pathologic relationship between the ACL and a narrow or stenosed notch because of direct impingement of the notch on the ACL during hyperextension or contact between the medial aspect of the

OrthoPedics and Ossur; receives publishing royalties, financial or material support from Saunders/Mosby-Elsevier and Wolter Kluer Health - Lippincott Williams $\theta$ Wilkins. Full ICMJE author disclosure forms are available for this article online, as supplementary material.

Received October 4, 2020; accepted January 27, 2021.

Address correspondence to Robert L. Parisien, M.D., Harvard Medical School and Boston Children's Hospital, 300 Longwood Avenue, Boston, MA 02115,U.S.A.E-mail:Robert.L.Parisien@gmail.com

(C) 2021 THE AUTHORS. Published by Elsevier Inc. on behalf of the Arthroscopy Association of North America. This is an open access article under the CC BY-NC-ND license (http://creativecommons.org/licenses/by-nc-nd/4.0/).

2666-061X/201645

https://doi.org/10.1016/j.asmr.2021.01.024 
lateral femoral condyle and the ACL during rotational activities such as cutting or pivoting. ${ }^{10-15}$

In 1988, Souryal and colleagues ${ }^{16}$ introduced the concept of the notch width index (NWI) in an attempt to account for and standardize anatomic variations in the physical size differences of patients' knees. The NWI is determined as the ratio of the intercondylar notch to the bicondylar width of the femur at the level of the popliteal groove. Previous studies have demonstrated an association between a smaller NWI (narrower notch) and an increased risk of ACL rupture in the adult population. ${ }^{17-19}$ However, there is limited and conflicting evidence in the pediatric population. In our opinion, the degree of controversy in this particular patient population warrants further analysis of NW morphology as a risk factor for ACL injury in children and adolescents.

The purposes of this study were to evaluate the NWI as a risk factor for ACL rupture in the pediatric and adolescent patient population via multicenter analysis and to detect any differences in the NWI among cohorts having sustained either a unilateral or bilateral ACL rupture. We hypothesized that a smaller NWI would prove to be a risk factor for both unilateral and bilateral ACL injuries as compared to uninjured controls.

\section{Methods}

This was an institutional review board-approved retrospective study conducted at 2 academic pediatric orthopaedic tertiary-care hospitals. In an attempt to minimize confounding, a multicenter case control analysis was performed with a consecutive series of patients enrolled from January 1999 through July 2010.

The study was designed around 3 cohorts: unilateral ACL rupture group, bilateral ACL rupture group, and an uninjured control group. Inclusion criteria consisted of patients 10-18 years of age at the time of diagnosis of a primary ACL tear (unilateral or bilateral) who underwent subsequent ACL reconstruction. All patients underwent preoperative magnetic resonance imaging (MRI) and at least 6 months of postoperative follow-up. All ACL re-ruptures were excluded from the study. Controls were determined as those patients having undergone previous MRI evaluation for knee pain without evidence of ACL pathology or a history of ACL rupture, fracture, or other diagnosis that may confound measurements of NWI.

\section{Notch Width Measurement}

One reviewer, blinded to the patient's clinical history, determined NW measurements via coronal MRI sequences. Each measurement was determined on 2 separate occasions more than 24 hours apart with the reviewer blinded to the initial measurement. The NWI measurements were determined in a standardized manner utilizing the technique described by Davis et al. ${ }^{20}$ The particular coronal sequence image chosen for measurement was the sequence in which the ACL and posterior cruciate ligament (PCL) are identified to cross each other closest to the mid-substance of the ACL. Typically in an uninjured knee, this cut is immediately anterior to the appearance of the roof of the intercondylar notch, with the ACL visualized longitudinally as the PCL is seen traversing posterior. The cut immediately posterior to the chosen image should have no visible notch roof and a more prominent distal PCL, and it should be near the ACL femoral attachment. The cut immediately anterior to the chosen image depicts the ACL near its tibial attachment and the PCL near its femoral attachment.

With selection of the appropriate image, the intercondylar NW was measured at the midpoint of the notch height (Fig 1A). This is identified where the ACL and PCL would cross one another halfway between the notch apex and the articular surface of the medial femoral condyle. The femoral bicondylar width was then measured at the same level as the NW (Fig 1B). The NWI was calculated as the NW divided by the femoral bicondylar width. For patients in the bilateral rupture group, the NWI was only measured in the initial injured knee to preserve the principles of independence.

\section{Statistical Analysis}

Univariate analysis was performed via $\chi^{2}$ test for categorical variables (e.g., sex). Analysis of variance and subsequent 1- or 2-tailed Student's $t$-rests were used for continuous variables (i.e., age and NWI), as appropriate, using Stata (Version 12.1; StataCorp) after normality of data was determined with both ShapiroWilk and Kolmogorov-Smirnov tests. Additional analysis was done after combining the unilateral and bilateral rupture cohort into a single "ACL injury" group with direct comparison to the control cohort.

\section{Results}

Of the 68 patients included for analysis, 22 sustained unilateral ACL rupture, 23 sustained bilateral ruptures and 23 comprised the control group. There was no difference in age or sex among the groups. The patient characteristics are represented in Table 1.

The NWI measurements calculated in all 3 cohorts were normally distributed with an analysis of variance calculation identifying a statistically significant difference in NWI among the 3 groups with $P=.015$. The intrarater reliability for NWI measurements was strongly positive with $r=0.96$. In direct comparison of the bilateral rupture group (mean $0.240 \pm 0.019$ ) and the control group (mean $0.262 \pm 0.029$ ), there was a statistically significant difference appreciated in NWI with $P=.002$. A statistically significant difference was also realized in direct comparison of the unilateral rupture (mean $0.248 \pm 0.026$ ) and control groups $(0.262 \pm 0.029)$ with $P=.047$ (Fig 2). In direct 


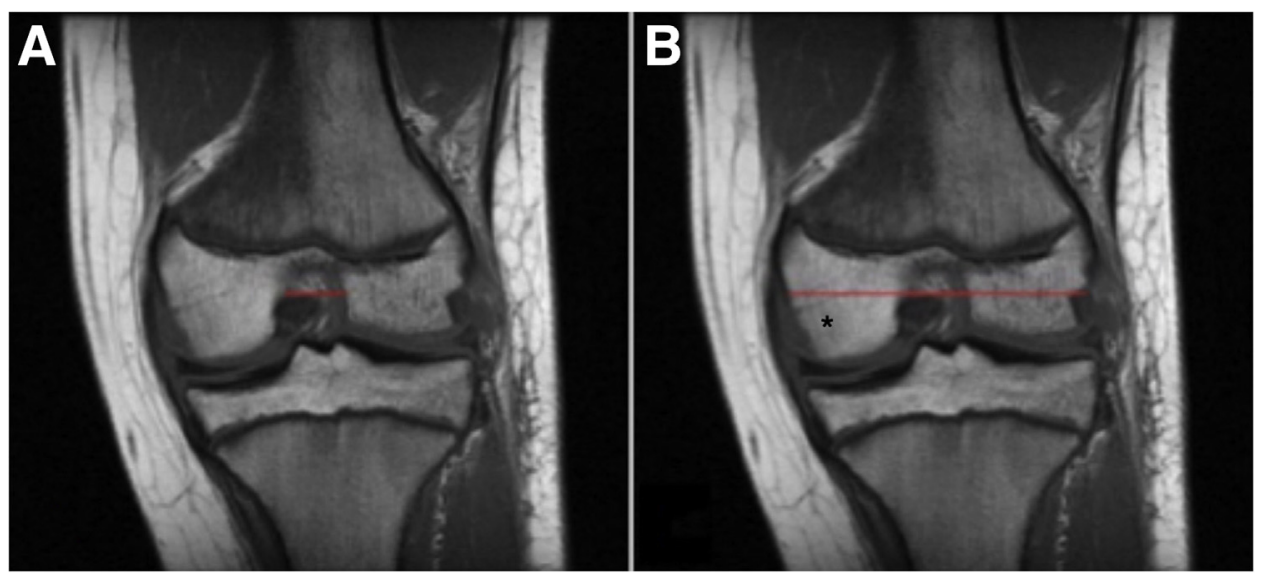

Fig 1. (A) Intercondylar notch width measurement. Intercondylar notch width (red line) is measured on a coronal T2-weighted magnetic resonance imaging (MRI) sequence at the mid-point of the notch height, where the anterior cruciate ligament (ACL) and posterior cruciate ligament would cross one another halfway between the notch apex and the articular surface of the medial femoral condyle. (B) Bicondylar width measurement. Bicondylar distal femoral width (red line) is measured on a coronal T2-weighted MRI sequence at the same level as the notch width measurement (A) spanning from the medial cortex of the medial femoral condyle $\left(^{*}\right)$ to the lateral cortex of the lateral femoral condyle.

comparison of the unilateral rupture group to the bilateral rupture group, there was no statistically significant difference determined with regard to NWI.

Further subanalysis of cohorts comparing the combined ACL rupture group (unilateral and bilateral groups) to the control group demonstrated a statistically significant difference with a smaller NWI realized in the ACL rupture group (mean $0.244 \pm 0.023$ ) as compared to the control group (mean $0.262 \pm 0.029$ ) with $P=.003$ (Fig 2). These findings demonstrate that a smaller NWI is associated with both unilateral and bilateral ACL ruptures.

\section{Discussion}

In this case-control multicenter analysis at 2 academic pediatric orthopaedic hospitals, we identified a small NWI (narrower notch) to be significantly associated with primary ACL rupture in the pediatric and adolescent patient population. This finding provides further support in favor of the careful evaluation of nonmodifiable risk factors in the pediatric patient population.

Modifiable parameters have long been investigated with regard to ACL injury risk with robust analysis of neuromuscular function and biomechanics. ${ }^{21}$ Decreased neuromuscular control of the trunk, lower extremity

Table 1. Patient Characteristics

\begin{tabular}{lccc}
\hline & $\begin{array}{c}\text { Bilateral ACL } \\
\text { Rupture }\end{array}$ & $\begin{array}{c}\text { Unilateral ACL } \\
\text { Rupture }\end{array}$ & $\begin{array}{c}\text { Control } \\
\text { Group }\end{array}$ \\
\hline Total Patients & 23 & 22 & 23 \\
Male & 6 & 10 & 10 \\
Female & 17 & 12 & 13 \\
Age (mean) & 15.10 & 14.52 & 14.48 \\
NWI (mean) & 0.240 & 0.248 & 0.262 \\
\hline
\end{tabular}

ACL, anterior cruciate ligament; NWI, notch width index. propioception, and increased dynamic valgus loads have been shown to be risk-factors for ACL injury in female athletes in particular. ${ }^{3,4}$ Other modifiable biomechanical risk factors include increased knee abduction upon landing, decreased lower extremity muscular strength and proprioception, and an imbalance of neuromuscular and ligament control among female athletes. ${ }^{22}$

In comparison, nonmodifiable risk factor analysis has recently grown in the adult patient population to better understand the complex multifactorial nature of ACL injury. Morphologic anatomic characteristics of the knee have specifically garnered significant investigative interest, with studies evaluating both femoral and tibial parameters. ${ }^{8,9,23}$ Such analyses have included comprehensive evaluation of the variation in femoral notch and condylar morphology. In the adult population, decreased femoral NW and NWI have been shown to increase the risk for both primary ACL injury and secondary contralateral ACL injury. ${ }^{24,25}$ Patients with elevated body mass index and a smaller femoral NW have been shown to be especially vulnerable to ACL injury. ${ }^{26}$ Other studies have found the intercondylar notch volume to correlate with ACL volume and that decreased intercondylar NW at both the inlet and outlet are associated with ACL injury. ${ }^{27}$ Furthermore, a decreased intercondylar notch angle has been reported to be associated with ACL tears. ${ }^{28}$ Interestingly, analysis of femoral condyle morphology and dimension has demonstrated gender-related differences and inconsistent findings. One study found that differences in medial condyle width, lateral condyle width, and bicondylar width were associated with ACL rupture in female patients whereas another study did not appreciate a difference in the shape or size of either condyle. ${ }^{29-31}$ 


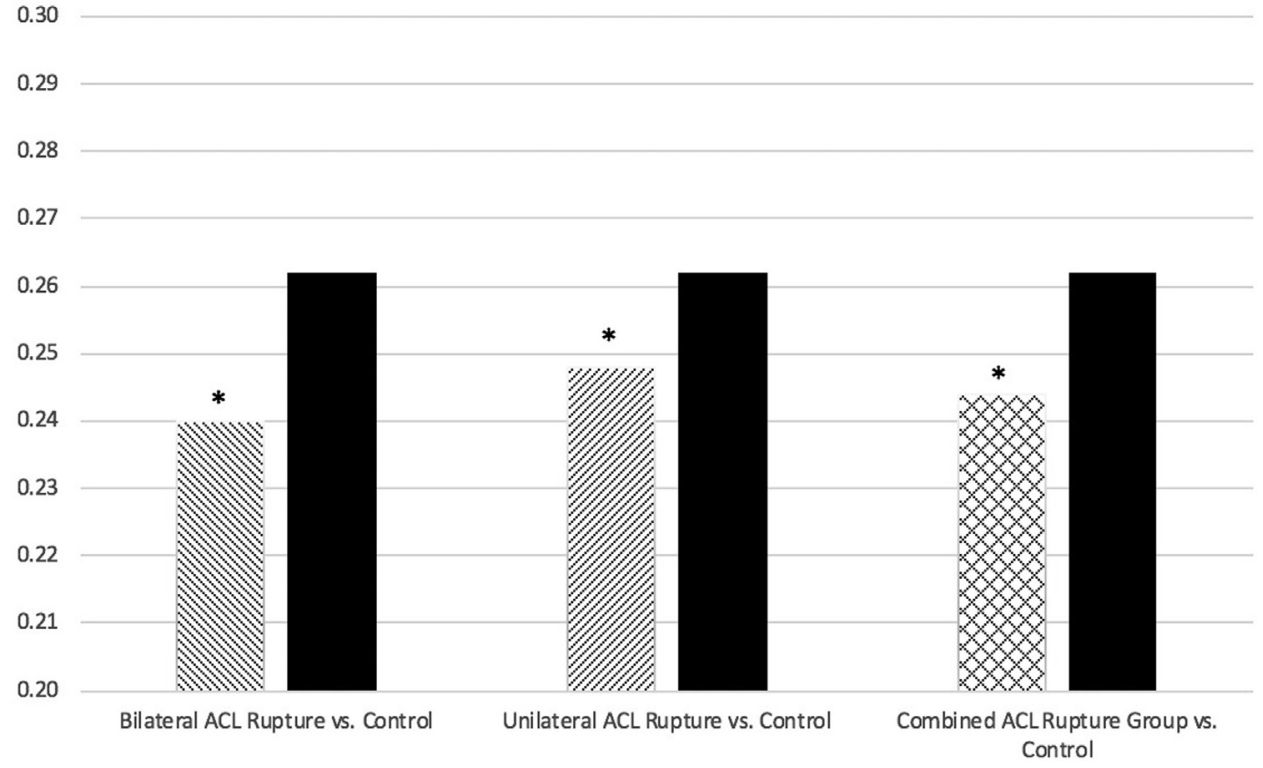

Fig 2. Comparison of notch width index (NWI) between groups. In direct comparison between the bilateral anterior cruciate ligament (ACL) rupture group versus the control group, the unilateral ACL rupture group versus the control group, and the combined ACL rupture group (both unilateral and bilateral group) versus control group, statistically significant differences were appreciated in NWI measurements. *Statistically significant $(P<.05)$.
Similarly, morphologic analyses relating to the tibia have included comprehensive evaluation of the variation in tibial slope, tibial eminences, subchondral tibial bone plate, and meniscus integrity. A decrease in the height of the tibial spine, the width of the lateral tibial spine, and the thickness of the medial tibial compartment articular cartilage have been identified as having an increased risk of secondary contralateral ACL injury in female athletes. ${ }^{25}$ An increased medial and lateral posterior tibial slope was also found to be associated with ACL tears in male patients specifically. ${ }^{28,32}$ However, additional analyses demonstrate a lack of association between medial tibial slope, posterior tibial slope, lateral tibial slope, or tibial insertion site length with ACL injury in either male or female patients. $^{30,33}$

Additional literature has demonstrated variable findings in attempt to evaluate nonmodifiable risk factors with particular focus on anatomic analysis of tibiofemoral congruity and Q-angle measurements. One study reported no association between the Q-angle and risk of ACL injury, whereas an additional study reported a modest difference in the Q-angle between patients with and without ACL injury. ${ }^{29,34}$ Furthermore, decreased tibial plateau anteroposterior (AP) distances and a higher ratio of the AP-flattened surface of the lateral femoral condyle to the tibial plateau AP distance was found to be associated with an increased risk of ACL injury in both male and female patients. ${ }^{35,36} \mathrm{An}$ increased convexity of the articulating surfaces of the proximal tibia and distal femur has also been shown to increase the risk of ACL injury. ${ }^{36}$ However, a separate study by Chung et al. ${ }^{37}$ found no association between the tibiofemoral angle and risk for ACL injury.
Distinguishable from tibial slope analysis, which is thought to increase ACL strain during flexion, a narrow notch is thought to directly impinge upon the ACL during hyperextension or during contact between the medial aspect of the lateral femoral condyle and the ACL during rotational activities such as cutting or pivoting, ${ }^{10-15}$ thus contributing to increased ACL tension leading to rupture. The vast majority of studies evaluating morphologic anatomic risk factors have taken place in the adult population and largely consist of cohorts from a single-institution. In contrast, our study is comprised of data collected at 2 leading academic pediatric hospitals with particular focus on the pediatric and adolescent patient population. Furthermore, we analyzed the association between NWI and ACL rupture for patients with both unilateral and bilateral tears as compared to controls without underlying ACL pathology. The existing literature within this domain has demonstrated rather inconsistent results with regard to the contribution of NWI to ACL injury risk. Domzalski et al. ${ }^{24}$ reported a significant association between NWI and ACL injury within an age-matched adolescent population whereas Swami et al. ${ }^{38}$ found no association between NWI and ACL injury risk. Therefore our evaluation of 68 pediatric patients across 2 academic pediatric hospitals is an important contribution to the limited literature analyzing the association between femoral NW and ACL injury risk.

\section{Limitations}

However, this study is not without limitation. The retrospective design is inherently susceptibility to selection bias. However, the enrollment of consecutive patients across 2 academic pediatric hospitals was conducted 
in attempt to mitigate the impact of any selection bias that may be present. Additionally, although the measurements of NWI demonstrated excellent intrarater reliability, an additional blinded reviewer would afford the opportunity to determine interrater reliability.

Although there was a statistically significant difference in NWI determined between the ACL injury groups and the uninjured control group, further studies may prove useful in exploring the determination of a specific NWI threshold value that may help guide injury risk prevention protocols in the pediatric and adolescent athlete. Future analyses may seek to investigate sex-specific differences with regard to an identified NWI threshold to further enhance targetted injury prevention programs.

\section{Conclusion}

Given the known inconsistencies in the existing literature, our findings provide further support of a narrow NWI as a significant contributing factor to both unilateral and bilateral ACL injury risk in the pediatric and adolescent population.

\section{References}

1. Dodwell ER, LaMont LE, Green DW, Pan TJ, Marx RG, Lyman S. 20 Years of Pediatric Anterior Cruciate Ligament Reconstruction in New York State. Am J Sports Med 2014;42:675-680.

2. Werner BC, Yang S, Looney AM, Gwathmey FWJ. Trends in pediatric and adolescent anterior cruciate ligament injury and reconstruction. J Pediatr Orthop 2016;36: 447-452.

3. Hewett TE, Myer GD, Ford KR, et al. Biomechanical measures of neuromuscular control and valgus loading of the knee predict anterior cruciate ligament injury risk in female athletes: A prospective study. Am J Sports Med 2005;33:492-501.

4. Zazulak BT, Hewett TE, Reeves NP, Goldberg B, Cholewicki J. Deficits in neuromuscular control of the trunk predict knee injury risk: Prospective biomechanicalepidemiologic study. Am J Sports Med 2007;35:1 123-1130.

5. Shea KG, Pfeiffer R, Wang JH, Curtin M, Apel PJ. Anterior cruciate ligament injury in pediatric and adolescent soccer players: An analysis of insurance data. J Pediatr Orthop 2004; $24: 623-628$.

6. Hewett TE, Myer GD, Ford KR. Anterior cruciate ligament injuries in female athletes: Part 1, mechanisms and risk factors. Am J Sports Med 2006;34:299-311.

7. Myer GD, Ford KR, Paterno MV, Nick TG, Hewett TE. The effects of generalized joint laxity on risk of anterior cruciate ligament injury in young female athletes. Am J Sports Med 2008;36:1073-1080.

8. Vyas S, van Eck CF, Vyas N, Fu FH, Otsuka NY. Increased medial tibial slope in teenage pediatric population with open physes and anterior cruciate ligament injuries. Knee Surg Sports Traumatol Arthrosc 2011;19:372-377.

9. Dare DM, Fabricant PD, McCarthy MM, et al. Increased lateral tibial slope is a risk factor for pediatric anterior cruciate ligament injury: An MRI-based case-control study of 152 patients. Am J Sports Med 2015;43:1632-1639.

10. Shelbourne KD, Facibene WA, Hunt JJ. Radiographic and intraoperative intercondylar notch width measurements in men and women with unilateral and bilateral anterior cruciate ligament tears. Knee Surg Sports Traumatol Arthrosc 1997; 5:229-233.

11. Norwood LA, Cross MJ. The intercondylar shelf and the anterior cruciate ligament. Am J Sports Med 1977;5: $171-176$.

12. Feagin JA, Lambert KL. Mechanism of injury and pathology of anterior cruciate ligament injuries. Orthop Clin North Am 1985;16:41-45.

13. Harner CD, Paulos LE, Greenwald AE, Rosenberg TD, Cooley VC. Detailed analysis of patients with bilateral anterior cruciate ligament injuries. Am J Sports Med 1994;22:37-43.

14. Kennedy JC, Weinberg HW, Wilson AS. The anatomy and function of the anterior cruciate ligament: As determined by clinical and morphological studies. J Bone Joint Surg Am $1974 ; 56: 223-235$.

15. LaPrade RF, Burnett QM. Femoral intercondylar notch stenosis and correlation to anterior cruciate ligament injuries: A prospective study. Am J Sports Med 1994;22: 198-203.

16. Souryal TO, Moore HA, Evans JP. Bilaterality in anterior cruciate ligament injuries: Associated intercondylar notch stenosis. Am J Sports Med 1988;16:449-454.

17. Hoteya K, Kato Y, Motojima S, et al. Association between intercondylar notch narrowing and bilateral anterior cruciate ligament injuries in athletes. Arch Orthop Trauma Surg 2011;131:371-376.

18. Sonnery-Cottet B, Archbold P, Cucurulo T, et al. The influence of the tibial slope and the size of the intercondylar notch on rupture of the anterior cruciate ligament. Bone Joint J 201 1;93-B:1475-1478.

19. Görmeli CA, Görmeli G, Öztürk YB, Özdemir Z, Kahraman A. The effect of the intercondyler notch width index on anterior cruciate ligament injuries: A study on groups with unilateral and bilateral ACL injury. Orthop $J$ Sports Med 2014;2(11_suppl3):2325967114S2325900204.

20. Davis TJ, Shelbourne KD, Klootwyk TE. Correlation of the intercondylar notch width of the femur to the width of the anterior and posterior cruciate ligaments. Knee Surg Sports Traumatol Arthrosc 1999;7:209-214.

21. Hewett TE, Myer GD, Ford KR, Paterno MV, Quatman CE. Mechanisms, prediction, and prevention of ACL injuries: Cut risk with three sharpened and validated tools. J Orthop Res 2016;34:1843-1855.

22. Myer GD, Ford KR, Hewett TE. Rationale and clinical techniques for anterior cruciate ligament injury prevention among female athletes. J Athl Train 2004;39:352-364.

23. Bayer S, Meredith SJ, Wilson K, et al. Knee morphological risk factors for anterior cruciate ligament injury: A systematic review. J Bone Joint Surg Am 2020;102:703-718.

24. Domzalski M, Grzelak P, Gabos P. Risk factors for anterior cruciate ligament injury in skeletally immature patients: analysis of intercondylar notch width using magnetic resonance imaging. Int Orthop 2010;34:703-707.

25. Levins JG, Argentieri EC, Sturnick DR, et al. Geometric characteristics of the knee are associated with a 
noncontact acl injury to the contralateral knee after unilateral ACL injury in young female athletes. Am J Sports Med 2017;45:3223-3232.

26. Evans KN, Kilcoyne KG, Dickens JF, et al. Predisposing risk factors for non-contact ACL injuries in military subjects. Knee Surg Sports Traumatol Arthrosc 2012;20: 1554-1559.

27. Simon RA, Everhart JS, Nagaraja HN, Chaudhari AM. A case-control study of anterior cruciate ligament volume, tibial plateau slopes and intercondylar notch dimensions in ACL-injured knees. J Biomech 2010;43:1702-1707.

28. Alentorn-Geli E, Pelfort X, Mingo F, et al. An evaluation of the association between radiographic intercondylar notch narrowing and anterior cruciate ligament injury in men: The notch angle is a better parameter than notch width. Arthroscopy 2015;31:2004-2013.

29. Kızılgöz V, Sivrioğlu AK, Ulusoy GR, Aydın H, Karayol SS, Menderes U. Analysis of the risk factors for anterior cruciate ligament injury: An investigation of structural tendencies. Clin Imaging 2018;50:20-30.

30. van Diek FM, Wolf MR, Murawski CD, van Eck CF, Fu FH. Knee morphology and risk factors for developing an anterior cruciate ligament rupture: an MRI comparison between ACL-ruptured and non-injured knees. Knee Surg Sports Traumatol Arthrosc 2014;22(5):987-994.

31. Siebold R, Axe J, Irrgang JJ, Li K, Tashman S, Fu FH. A computerized analysis of femoral condyle radii in ACL intact and contralateral ACL reconstructed knees using 3D CT. Knee Surg, Sports Traumatol Arthrosc 2010;18:26-31.
32. Rahnemai-Azar AA, Yaseen Z, van Eck CF, Irrgang JJ, $\mathrm{Fu}$ FH, Musahl V. Increased lateral tibial plateau slope predisposes male college football players to anterior cruciate ligament injury. J Bone Joint Surg Am 2016;98: 1001-1006.

33. Lopes OV, Gomes JLE, de Freitas Spinelli L. Range of motion and radiographic analysis of the hip in patients with contact and non-contact anterior cruciate ligament injury. Knee Surg Sports Traumatol Arthrosc 2016;24:2868-2873.

34. Hertel J, Dorfman JH, Braham RA. Lower extremity malalignments and anterior cruciate ligament injury history. J Sports Sci Med 2004;3:220-225.

35. Vasta S, Andrade R, Pereira R, et al. Bone morphology and morphometry of the lateral femoral condyle is a risk factor for ACL injury. Knee Surg Sports Traumatol Arthrosc 2018;26:2817-2825.

36. Wahl CJ, Westermann RW, Blaisdell GY, Cizik AM. An association of lateral knee sagittal anatomic factors with non-contact ACL injury: Sex or geometry? J Bone Joint Surg Am 2012;94:217-226.

37. Chung SC-Y, Chan W-L, Wong S-H. Lower Limb alignment in anterior cruciate ligament-deficient versus -intact knees. J Orthop Surg 2011;19:303-308.

38. Swami VG, Mabee M, Hui C, Jaremko JL. Three-dimensional intercondylar notch volumes in a skeletally immature pediatric population: A magnetic resonance imaging-based anatomic comparison of knees with torn and intact anterior cruciate ligaments. Arthroscopy 2013;29:1954-1962. 